\title{
Cannabis sativa var. indica: une menace croissante pour les entreprises
}

\section{Cannabis sativa var. indica : an increasing hazard for firms}

Patrick MURA*

Laboratoire de Biochimie et de Toxicologie, Centre Hospitalier Universitaire = BP 577 - 86021 POITIERS Cedex

* Auteur à qui adresser la correspondance : Patrick MURA, Laboratoire de Biologie et de Toxicologie, Centre Hospitalier Universitaire - BP 577 - 86021 POITIERS Cedex Tél : 0549443923 - Fax : 0549443834 - e-mail : p.mura@chu-poitiers.fr

(Reçu le $1^{e r}$ février 2002 ; accepté le 15 février 2002)

\section{RÉSUMÉ}

Parmi les drogues illicites, le cannabis est de très loin le produit le plus consommé. Les effets sur l'organisme sont principalement dus au delta-9-tétrahydrocannabinol (THC). Lors d'un usage peu important et occasionnel, ils consistent en une euphorie, une désinhibition, un état de somnolence, avec détérioration de la perception temporelle et spatiale. Les perturbations de la vision consistent en une mydriase, pouvant être accompagnée d'un nystagmus et d'une diplopie. Des troubles de la mémoire à court terme sont fréquemment observés. Ces effets, qui persistent pendant 2 à 10 heures, sont difficilement compatibles avec la réalisation d'actes complexes. Un usage fréquent et important peut conduire à des attaques de panique, des crises d'angoisse, voire l'apparition d'une psychose cannabique avec notamment la survenue d'hallucinations visuelles. C'est en raison de ces effets que son usage est considéré comme étant incompatible avec une conduite automobile en toute sécurité. Une étude française récente, réalisée chez 900 conducteurs impliqués dans un accident corporel de la voie publique et 900 sujets témoins, a montré que le nombre des accidents était multiplié par 2,5 chez les conducteurs ayant consommé du cannabis dans les heures précédentes. Aussi la législation française permet désormais de dépister son usage chez les conducteurs impliqués dans un accident corporel de la circulation, avec une obligation dans le cas des accidents mortels. L'absence de récepteurs au niveau bulbai-

\section{SUMMARY}

Among the various drugs of abuse, cannabis is by far the most consumed. The effects on human organism are mainly due to delta-9 tetrahydrocannabinol (THC). With low doses and infrequent use, the effects are euphoria, desinhibition, drowsiness, and an alteration of temporal and spatial perception. Visual disorders consist in a mydriasis sometimes accompanied with a nystagmus and a diplopia. Short-memory troubles are frequently observed. These effects, which can persist during 2 to 10 hours, are not compatible with the realization of complex activities. A frequent use may lead to panic and anxiety attacks, and psychiatric disorders such as psychosis with visual hallucinations. Because of these effects, its use is considered to affect traffic safety. A recent French study, performed on 900 drivers involved in a corporal accident and 900 control subjects, indicated the number of accidents was multiplied by 2.5 when drivers had smoked cannabis a few hours before. So, a French law allows to identify drug users in drivers involved in a corporal car accident, with an obligation in the case of fatal accidents. The lack of receptors in the bulb, and then the non-occurrence of deaths by overdose, led some people to the conclusion that cannabis is the least dangerous of addict products. Such an assertion disregards the important functional neurotoxicity of THC which is the reason why cannabis use may represent 
re, et ce faisant l'absence de décès par overdose, a conduit certaines personnes à classer ce produit parmi les substances addictives les moins dangereuses pour l'homme. Une telle classification fait abstraction de la neurotoxicité fonctionnelle importante du THC qui fait que l'usage de ce produit peut constituer un facteur de risque pour autrui. Cela est vrai pour la conduite automobile. Cela peut être tout aussi vrai lorsque les consommateurs occupent des postes de travail dans lesquels les fautes professionnelles peuvent mettre en péril l'entreprise et/ou la collectivité. Ce risque était négligeable à une époque où la consommation de cannabis était marginale. Il devient majeur aujourd'hui compte tenu du nombre de consommateurs et des mesures de prévention s'imposent désormais, au delà du seul principe de précaution.

\section{MOTS-CLÉS}

Cannabis, effets, poste de travail.

\section{Introduction}

Les effets neuropsychiques recherchés par les consommateurs de cannabis sont essentiellement dus, lorsque le cannabis est inhalé, au delta-9 tétrahydrocannabinol (THC). Les effets chez l'homme de l'usage de cannabis ont été explorés par de nombreux travaux (1). En plus d'un certain nombre d'effets somatiques, ils consistent essentiellement en une altération des compétences cognitives et psychomotrices. Dans de nombreux pays, la connaissance de cette toxicité neurocomportementale a été à l'origine de la mise en place de mesures réglementaires visant à sanctionner la conduite d'un véhicule à moteur sous l'emprise de cannabis (2). En France, un texte de loi a été promulgué en ce sens en 1999 (3), dont les décrets d'application ont tout récemment été publiés $(4,5)$. Une étude épidémiologique récente a montré que, chez les moins de 27 ans, la fréquence des accidents corporels était multipliée par 2,5 avec le cannabis lorsqu'il était consommé seul et par 4,8 lorsqu'il était consommé en association avec de l'alcool (6). S'il a été ainsi démontré que l'aptitude à conduire un véhicule en toute sécurité était largement altérée par un usage récent de cannabis, qu'en est-il des conséquences de cette consommation chez des sujets qui occupent des postes de travail dans lesquels les fautes professionnelles peuvent mettre en péril l'entreprise et/ou la collectivité ? Est-ce un risque négligeable ? Un sujet ayant fumé un ou deux «joints» la veille au soir est-il encore un danger pour l'entreprise le lendemain ? Dans cet article, nous nous attacherons à répondre à toutes ces questions en nous efforçant de n'utiliser comme argumentation que les données validées par la communauté scientifique internationale.

\section{Le cannabis est la drogue illicite la plus consommée.}

a risk factor for others. That is true for car driving. That may be also true in factories when consumers occupy operation stations in which professional errors may imperil the enterprise and/or the collectivity. This risk was negligible when cannabis consumption was rare. But it is very important today on account of the number of consumers and so, prevention measures are now necessary.

\author{
KEY-WORDS \\ Cannabis, effects, workstation.
}

A ce jour en France, plus de $20 \%$ des personnes de 15 à 75 ans ont expérimenté le cannabis tandis que 5,5\% d'entre eux ont expérimenté une autre drogue illicite (7). Depuis 1990, cette consommation est en constante augmentation, notamment chez les jeunes. Chez les jeunes de 18 ans, la prévalence-vie (personnes ayant consommé au moins une fois dans la vie) est passé de $7 \%$ en 1992 à $23 \%$ en 1996, puis $34 \%$ et $39 \%$ en 1998 et 2000 (1). Les résultats d'une enquête récente indiquent que, entre 1993 et 1999, la consommation répétée (au moins dix fois dans l'année) a augmenté chez les garçons de 18 ans de 11 à $29 \%$ et de 3 à $14 \%$ chez les filles du même âge. Une autre enquête récente a montré que la proportion de jeunes de 18 ans avouant consommer du cannabis plus de 10 fois par an avait évolué considérablement de 1993 à 1999, passant de $3 \%$ à $14 \%$ chez les filles et de 11 à $29 \%$ chez les garçons (8). Bien qu'à un degré moindre, cette tendance concerne aussi les adultes. Entre 1995 et 2000, dans la tranche d'âge des 25-29 ans, la prévalence-vie est passée de $5 \%$ à $13 \%$ (9). Il n'est donc pas surprenant que le cannabis soit le produit le plus fréquemment rencontré lors des dépistages de toxicomanie en entreprise (10).

\section{Le cannabis a des effets extrêmement variés}

Une des particularités du cannabis est la grande variété des effets. Ils sont variables selon la quantité consommée, la qualité du produit (11), la tolérance du sujet, la structure de sa personnalité et son état d'esprit du moment, le contexte dans lequel s'inscrit la consommation et bien entendu selon qu'il a été pris isolément ou associé à d'autres produits psychotropes (autres drogues, alcool, médicaments). 


\section{Lors d'un usage occasionnel, les effets du cannabis sont principalement (1) :}

- des perturbations sensorielles : perception exacerbée des sons et surtout des modifications de la vision associées à une mydriase, une diplopie et un nystagmus,

- des troubles thymiques et dissociatifs avec euphorie, anxiété, agressivité, dépersonnalisation avec disparition des inhibitions et indifférence vis-à-vis de l'environnement, une conscience accrue de soi,

- des hallucinations et délires exceptionnels mais possibles, notamment avec les nouveaux produits très concentrés en cannabinoïdes,

- une diminution des performances intellectuelles (baisse de la productivité et de la concentration avec une pensée fragmentaire), motrices et cognitives,

- des modifications de la perception du temps et des distances,

- des perturbations de la mémoire à court terme.

On peut noter aussi une décompensation psychotique se traduisant par un syndrome délirant organique dont le thème le plus fréquent est la persécution et associe anxiété, tremblements, incoordination motrice.

Lors d'un usage fréquent et prolongé de cannabis, des crises d'angoisse aiguë peuvent survenir au cours desquelles un véritable état de panique s'installe. Par ailleurs, un syndrome amotivationnel est classiquement observé chez les usagers chroniques. Généralement lié à une imprégnation cannabique importante et ancienne, il associe un déficit de l'activité, une indifférence affective, une altération du fonctionnement intellectuel et un ralentissement de la pensée. Un usage régulier et important de cannabis favoriserait la survenue de la schizophrénie. En effet l'association schizophrénie et addiction au cannabis est relativement fréquente, cette comorbidité allant jusqu'à $40 \%$ dans certaines études (12).

\section{Conséquences néfastes de l'utilisation du cannabis.}

Principale cause de sa banalisation, le cannabis a été classé par certains auteurs comme un des produits d'addiction les moins dangereux pour l'homme (13). Une telle classification reposait essentiellement sur l'absence de mortalité par surdosage, ce qui s'explique par l'absence de récepteurs au THC dans la région bulbaire et par l'absence de neurotoxicité cellulaire. Cependant, ce classement ne considérait que la dangerosité pour soi-même, faisant totalement abstraction des conséquences néfastes que son usage pouvait avoir pour autrui, du fait de la neurotoxicité fonctionnelle précédemment décrite.

\section{Baisse de productivité et absentéisme}

Le syndrome amotivationnel, la désinhibition voire les troubles psychotiques induits par un usage intense et régulier sont susceptibles d'augmenter l'absentéisme et d'entraîner, en milieu professionnel, une baisse de la productivité. Aux Etats-Unis, une étude a signalé des périodes plus longues et plus fréquentes de chômage chez les consommateurs de cannabis (14). Au Costa Rica, il a été montré que le nombre de chômeurs était plus important parmi les usagers (15).

\section{Accidents du travail et erreurs profes- sionnelles menaçant la vie d'autrui.}

Un ouvrier du bâtiment tombe d'un toit dont il changeait les tuiles. Un électricien professionnel expérimenté est électrocuté en installant une enseigne lumineuse. En restaurant un mur de pierre, un ouvrier est écrasé par l'effondrement de celui-ci. Un ouvrier agricole a un bras arraché par une machine outil. Dans tous ces cas d'accidents du travail, l'expertise toxicologique révélait la présence de THC dans le sang.

L'altération des performances cognitives et psychomotrices, les troubles de l'attention, de la mémoire immédiate et de la perception sensorielle induits par une consommation de cannabis suffisent largement pour expliquer ce risque accru d'accidents du travail.

Cependant, très peu d'études épidémiologiques ont été réalisées sur ce thème, certainement en raison du respect des droits individuels. Aussi, il est difficile de savoir qu'elle est, actuellement en France, la prévalence des accidents du travail imputables à une consommation antérieure de cannabis.

Les erreurs professionnelles responsables de blessures ou de morts et imputables à un usage récent de cannabis sont maintenant bien documentées dans le cadre de la conduite de véhicules à moteurs $(6,16)$. Il en est potentiellement de même pour toute activité professionnelle correspondant en l'accomplissement de tâches complexes (17).

\section{Durée des effets du cannabis}

Chez les gros consommateurs réguliers ayant développé des troubles psychiques comme le syndrome amotivationnel ou la schizophrénie, les altérations comportementales peuvent persister pendant plusieurs semaines voire quelques mois après arrêt de la consommation (1). Par ailleurs chez de tels sujets, le cannabis devient rapidement le centre de leurs préoccupations, ce qui semble être difficilement compatible avec un poste de travail nécessitant concentration et lucidité. Dans le cas d'une consommation irrégulière, la durée des effets psychiques est très variable selon les individus. Cette 
variabilité est principalement due à la manière de fumer et aux grandes variations du volume de distribution (4 à $14 \mathrm{~L} / \mathrm{kg}$ ). Très lipophile, le THC se distribue rapidement dans tous les tissus riches en lipides dont le cerveau. Cette forte fixation tissulaire, l'existence d'un cycle entéro-hépatique et de la réabsorption rénale se traduisent par des effets psychoactifs prolongés, pouvant persister dans le cas d'une consommation isolée jusqu'à 45 à 150 min après arrêt de la consommation (18). Johansson et coll (19) ont détecté des cannabinoïdes dans des biopsies de tissus adipeux 4 semaines après la dernière consommation de cannabis. Barnett et coll. (20) ont étudié, sur simulateurs de conduite, les effets (erreurs de conduite) obtenus par la consommation de «joints» dosés à 100, 200 ou $250 \mu \mathrm{g} / \mathrm{kg}$ de poids du sujet et ont indiqué l'existence d'une corrélation linéaire significative pendant une durée de 2 à $7 \mathrm{~h}$. Une étude effectuée sur simulateur de vol chez des pilotes d'avion chevronnés a mis en évidence une altération des performances psychomotrices des pilotes pouvant persister 24 heures après le moment de la consommation (21). Par ailleurs, il est à noter que des épisodes de «flash-back» ont été rapportés, au cours desquels le sujet revit tout ou partie de ces symptômes en l'absence de toute nouvelle consommation de cannabis. Ceci serait dû à un relargage brutal du THC accumulé dans les tissus adipeux.

En résumé et au vu de la littérature, un sujet ayant fumé 1 ou 2 «joints» la veille au soir pourrait représenter, le lendemain encore, un danger pour l'entreprise.

\section{Conclusion}

Conséquence d'une quasi-absence de prévention voire quelquefois de la promotion de ce produit, nous assistons depuis quelques années à une banalisation de la consommation de cannabis. Parmi les 3 millions de consommateurs occasionnels et les quelques 300.000 consommateurs quotidiens, la plupart conduisent un véhicule et certains d'entre eux occupent des postes de travail dans lesquels toute erreur peut avoir des conséquences pour eux-mêmes ou pour autrui. Or le THC se révèle être un produit psychoactif puissant, perturbant le fonctionnement des neurones, ce qui se traduit par une diminution des performances intellectuelles, motrices et cognitives. Dans le meilleur des cas, son influence sur l'organisme peut avoir comme conséquences une diminution de la productivité et une augmentation de l'absentéisme. Dans le pire des cas, les erreurs professionnelles peuvent aboutir au décès du consommateur ou d'autrui.

Face à cette menace croissante pour les entreprises, des mesures de prévention s'imposent désormais, au delà du seul principe de précaution.

\section{Références}

1. Expertise collective INSERM. Cannabis. Quels effets sur le comportement et la santé ? In : INSERM, ed. Paris : Jouve, 2001.

2. Charlier C., Verstraete A., Plomteux G. La législation dans les pays européens. In : Mura P. (coordinateur), Alcool, médicaments, stupéfiants et conduite automobile. Paris : Elsevier, $1999 ; 215-32$.

3. Journal Officiel de la République Française, 19 juin 1999, p. 9017.

4. Journal Officiel de la République Française, 28 août 2001, p. 13759.

5. Journal Officiel de la République Française, 18 septembre 2001, p. 14802.

6. Mura P., Gaulier J.M., Goullé J.P., Vincent F., Moulsma M., Kintz P. Prévalence de l'usage récent de psychotropes licites et illicites chez 900 conducteurs accidentés : comparaison avec 900 témoins. Ann. Toxicol. Anal. $2001 ; 13: 306$.

7. Drogues et dépendances : indicateurs et tendances de l'Observatoire Français des Drogues et des Toxicomanies. OFDT, rapport 2002.

8. Choquet M., Ledoux S., Hassler C., Beck F., Peretti-Watel P. Consommations de substances psychoactives chez les 14-18 ans scolarisés : premiers résultats de l'enquête ESPAD 1999, évolution 1993-1999. In : Observatoire Français des Drogues et des Toxicomanies, ed. Tendances, 2000, 6 .

9. Anonyme. Baromètre santé, enquête auprès des 12-75 ans, premiers résultats, CFES ed, 2000.

10. Verstraete A., Pierce A. Workplace drug testing in Europe. Forensic Sci. Int. $2001 ; 121$ : 2-6.

11. Mura P., Perrin M., Chabrillat M., Girard J., Dumestre-Toulet V., Pepin G. L'évolution des teneurs en THC dans les produits à base de cannabis en France : mythe ou réalité ? Ann. Toxicol. Anal. $2001 ; 13: 75-9$.

12. Kovasznay B., Fleischer J., Tanenberg-Karant M., Jandorf L., Miller A.D., Bromet E. Substance use disorder and the early course of illness in schizophrenia and affective psychosis. Schizophr. Bull. 1997 ; 23 : 195-201.

13. Roques B. La dangerosité des drogues. Rapport au secrétariat d'Etat à la santé. Paris : Odile Jacob / La documentation française, 1999.

14. Kandel D.B., Davies M. Labor force experiences of a national sample of young adult men. Youth and Society. $1990 ; 21$ : 411-45.

15. Carter W.E., Doughty P. Social and cultural aspects of cannabis use in Costa Rica. Ann. N. Y. Acad. Sci. 1976 ; 282 : 2-16.

16. Mura P., Piriou A. Le cannabis. In : Mura P. (coordinateur), Alcool, médicaments, stupéfiants et conduite automobile. Paris : Elsevier, 1999 ; 59-74.

17. Ameri A. The effects of cannabinoids on the brain. Prog. Neurobiol. $1999 ; 58$ : 315-48.

18. Stillman R., Galanter M., Lemberger L. Tetrahydrocannabinol (THC) : metabolism and subjective effects. Life Sci. $1976 ; 19$ : 569-76.

19. Johansson E., Noren K., Sjovall J., Halldin M.M. Determination of delta-1-tetrahydrocannabinol in human fat biopsies from marihuana users by gas chromatography-mass spectrometry. Biomed Chromatogr. $1989 ; 3$ : 35-8.

20. Barnett G., Licko V., Thompson T. Behavioral pharmacokinetics of marijuana. Psychopharmacology $1985 ; 85$ : 51-6.

21. Yesavage J.A., Leirer V.O., Denari M., Hollister L.E. Carry-over effects of marijuana intoxication on aircraft pilot performance : a preliminary report. Am J Psychiatry 1985 ; 142 : 1325-9. 